\title{
A patient with a germline SDHB mutation presenting with an isolated pituitary macroprolactinoma
}

\author{
Michelle Maher1,8, Federico Roncaroli2, Nigel Mendoza', Karim Meeran', Natalie Canham³, \\ Monika Kosicka-Slawinska4, Birgitta Bernhard4, David Collier5, Juliana Drummond5, Kassiani Skordilis6, \\ Nicola Tufton7, Anastasia Gontsarova', Niamh Martin', Márta Korbonits ${ }^{5}$ and Florian Wernig'
}

${ }^{1}$ Endocrinology, Imperial College Healthcare NHS Trust, London, UK, 2University of Manchester, Manchester, UK, ${ }^{3}$ Liverpool Womens NHS Foundation Trust, Liverpool, UK, 4London North West Healthcare NHS Trust, London, UK, 5The William Harvey Research Institute, Barts and The London School of Medicine and Dentistry, Queen Mary University of London, London, UK, 6 University Hospitals Birmingham NHS Foundation Trust, Mindelsohn Way, Edgbaston, Birmingham, UK, ${ }^{7}$ The Royal London Hospital, Barts Health NHS Trust, London UK and ${ }^{8}$ National University of Ireland, Galway, Ireland

Correspondence should be addressed to F Wernig

Email

f.wernig@imperial.ac.uk

\section{Summary}

Symptomatic pituitary adenomas occur with a prevalence of approximately $0.1 \%$ in the general population. It is estimated that $5 \%$ of pituitary adenomas occur in a familial setting, either in isolated or syndromic form. Recently, lossof-function mutations in genes encoding succinate dehydrogenase subunits (SDHX) or MYC-associated factor X (MAX) have been found to predispose to pituitary adenomas in co-existence with paragangliomas or phaeochromocytomas. It is rare, however, for a familial SDHx mutation to manifest as an isolated pituitary adenoma. We present the case of a pituitary lactotroph adenoma in a patient with a heterozygous germline SDHB mutation, in the absence of concomitant neoplasms. Initially, the adenoma showed biochemical response but poor tumour shrinkage in response to cabergoline; therefore, transsphenoidal surgery was performed. Following initial clinical improvement, tumour recurrence was identified 15 months later. Interestingly, re-initiation of cabergoline proved successful and the lesion demonstrated both biochemical response and tumour shrinkage. Our patient's SDHB mutation was identified when we realised that her father had a metastatic paraganglioma, prompting genetic testing. Re-inspection of the histopathological report of the prolactinoma confirmed cells with vacuolated cytoplasm. This histological feature is suggestive of an SDHx mutation and should prompt further screening for mutations by immunohistochemistry and/or genetic testing. Surprisingly, immunohistochemistry of this pituitary adenoma demonstrated normal SDHB expression, despite loss of SDHB expression in the patient's father's paraganglioma.

\section{Learning points:}

- Pituitary adenomas may be the presenting and/or sole feature of SDHB mutation-related disease.

- $S D H x$ mutated pituitary adenomas may display clinically aggressive behaviour and demonstrate variable response to medical treatment.

- Histological evidence of intracytoplasmic vacuoles in a pituitary adenoma might suggest an SDH-deficient tumour and should prompt further screening for SDHx mutations.

- Immunohistochemistry may not always predict the presence of SDHx mutations. 


\section{Background}

Symptomatic pituitary adenomas have a prevalence of approximately $0.1 \%$ in the general population. It is estimated that $5 \%$ of pituitary adenomas occur in a familial setting (1). In tumour predisposition syndromes like multiple endocrine neoplasia type 1 (MEN1) and type 4 (MEN4), and Carney complex they may develop in isolation or in association with other tumours (1). More recently, loss-of-function mutations in genes encoding succinate dehydrogenase $(\mathrm{SDH})$ subunits have been implicated in the development of the rare but increasingly recognised co-existence of paraganglioma/ phaeochromocytoma and pituitary adenoma $(2,3)$.

SDH constitutes the mitochondrial complex II. It is a heterotetrameric enzyme complex anchored to the inner mitochondrial membrane involved in both the Krebs cycle and electron transfer chain. Loss-of-function mutations in genes encoding the SDH subunits ( $\mathrm{SDH} x$ : $S D H A, S D H B, S D H C, S D H D)$ or its assembly factor (SDHAF2) have long been associated with various tumours, including paragangliomas and phaeochromocytomas, gastrointestinal stromal tumours (GISTs), renal carcinomas and pituitary adenomas. Previously, there have been three reported cases of pituitary adenoma occurring in association with familial $S D H B$ mutation, in the absence of a personal history of paraganglioma $(2,4)$. We describe a patient with $S D H B$ mutation who developed a lactotroph adenoma without any other tumour manifestation. The patient's father had a right carotid body paraganglioma with metastases.

\section{Case presentation}

A previously healthy 56-year-old postmenopausal woman presented with a 9-month history of bilateral visual loss. Bitemporal hemianopia was revealed on confrontation and was confirmed on Humphrey and Goldmann visual field perimetry, whilst cranial nerve examination was normal (Fig. 1A).

\section{Investigation}

MRI revealed a large pituitary mass harbouring a superior cystic component with extensive suprasellar and right cavernous sinus extension (Fig. 2A). Her serum prolactin measured 9675 (reference range: 100-500 mIU/L) with secondary suppression of gonadotrophins, LH $<0.5$ (>20 U/L) and FSH 1.8 (>20 U/L), supportive of a diagnosis of lactotroph adenoma. The rest of pituitary function
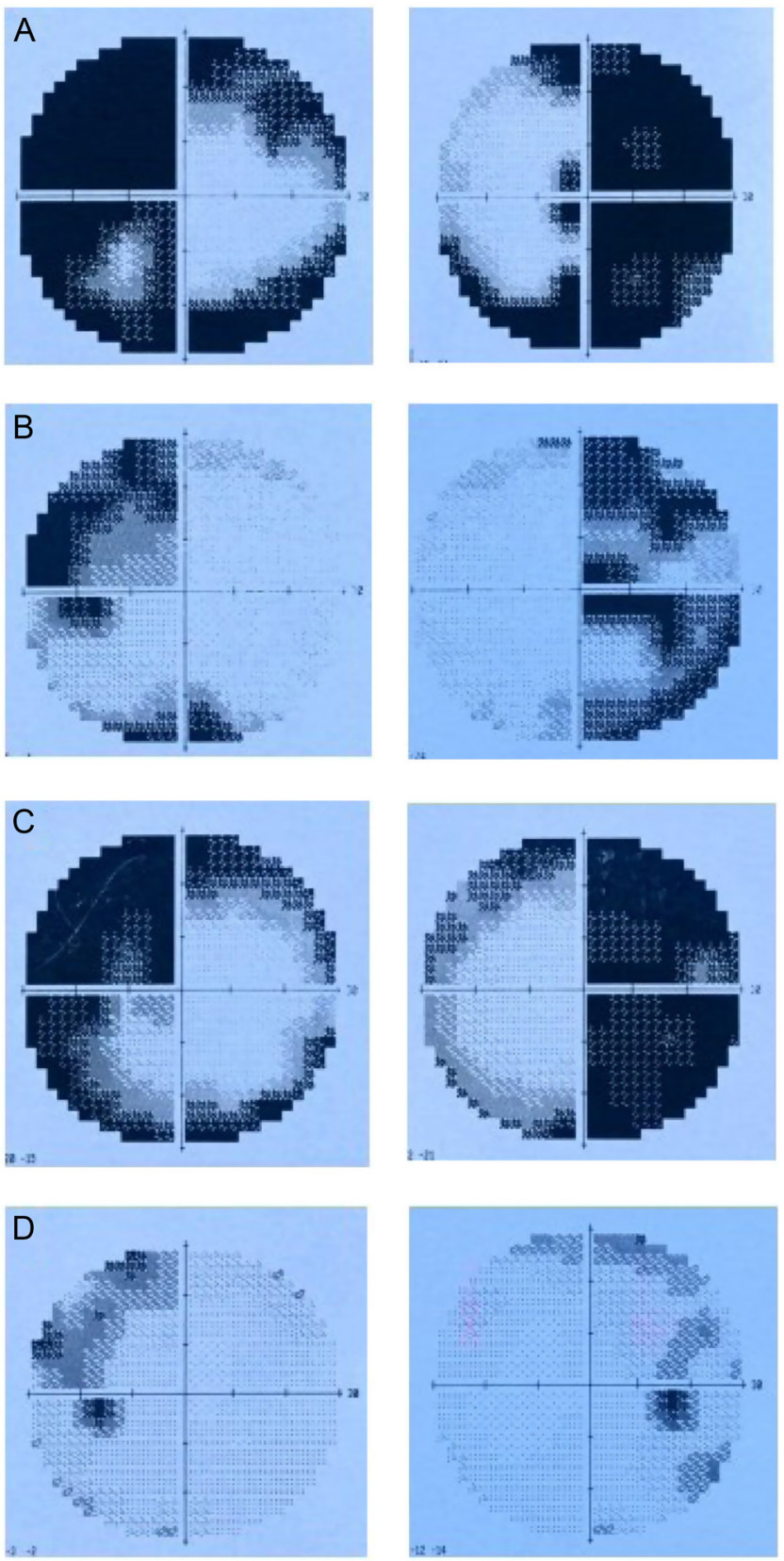

Figure 1

Visual field of the patient at diagnosis (A), on cabergoline treatment (B), before surgery (C) and after surgery (D).

was normal IGF1: $17.3(9-40 \mathrm{nmol} / \mathrm{L}), \mathrm{GH}<0.05 \mu \mathrm{g} / \mathrm{L}$, ACTH: 11.5 (09:00 h <30 ng/L), cortisol: 344 (09:00 h $160-550 \mathrm{nmol} / \mathrm{L})$, TSH: $1.06(0.3-4.2 \mathrm{mU} / \mathrm{L})$ and free T4: $16.4(9-23 \mathrm{pmol} / \mathrm{L})$.

\section{Treatment}

Cabergoline was commenced at a dose of $0.5 \mathrm{mg}$ weekly. Despite normalisation of serum prolactin at 1 month 

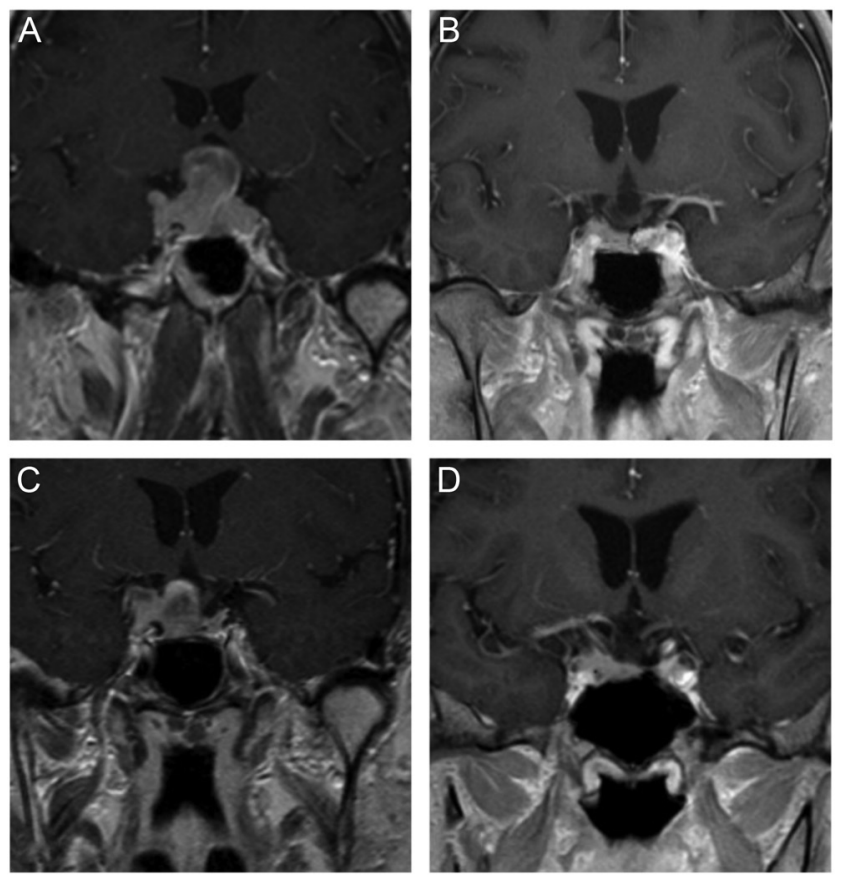

Figure 2

Preoperative contrast-enhanced T1 image (A) showing large pituitary mass with suprasellar and right cavernous sinus extension. Postoperative scan (B) showing small residual suprasellar tumour around the right carotid artery. Decompression and partial prolapse of the optic chiasm. MRI pituitary showing regrowth of the tumour 15 months after surgery (C) with suprasellar and right cavernous sinus extension and compression of the prolapsed optic chiasm. Significant shrinkage can be seen following 8 months cabergoline treatment (D) with disappearance of the suprasellar component.

(205 mIU/L), follow-up pituitary MRI (not shown) at 3 months showed minimal reduction in the size of the macroadenoma resulting in improvement in the visual field defect (Fig. 1B), but elevation of the optic chiasm and encroachment of the right cavernous sinus was present. Surgery was not pursued as her vision continued to improve and cavernous sinus involvement would have diminished the likelihood of curative surgery. Serial imaging over the next 18 months showed no further tumour size reduction. However, after 22 months, visual deterioration was noted (Fig. 1C) and the patient underwent transsphenoidal surgery. Histopathological examination of the resected tissue was consistent with a lactotroph adenoma. Some of the neoplastic cells demonstrated vacuolated cytoplasm (Fig. 3A). Approximately 20\% of adenoma cells were positive for prolactin, whilst immunostains for other pituitary hormones were negative. Nuclear expression of the transcription factor PIT-1 was intense and ubiquitous (Fig. 3B). The Ki-67 labelling index was less than 1\% and a few neoplastic cells demonstrated weak nuclear staining for p53. No mitosis was seen in the fragments examined (Trouillas classification 2a).
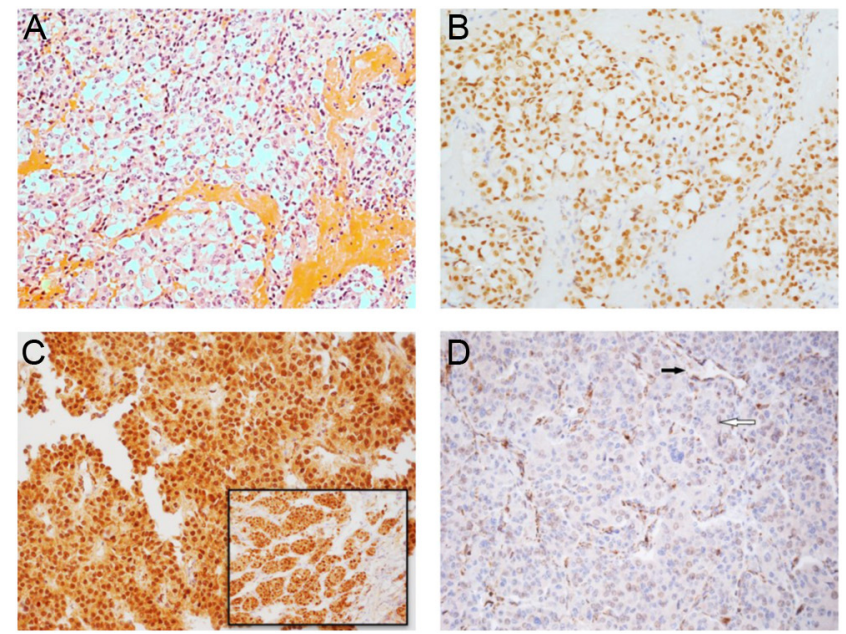

Figure 3

(A) Neoplastic cells demonstrate vacuolated cytoplasm (HE, $\times 40)$; (B) there is nuclear expression of Pit-1 (rabbit polyclonal, Novus Biological, Oxon, UK; dilution 1:100). (C) Neoplastic cells of the lactotroph adenoma express SDHB (Sigma, rabbit polyclonal, 1:150, immunoperoxidase, $\times 20$;

expression in the adjacent normal adenohypophysis is shown in the inset, $\times 40$ ); (D) SDHB expression in neoplastic cells of the carotid body paraganglioma from the patient's father is absent (immunoperoxidase, $\times 20$ ), black arrows indicate endothelial cells with normal SDHB expression while the white arrow indicates SDHB-negative tumour cells.

\section{Outcome and follow-up}

Hydrocortisone and levothyroxine replacement was initiated post-operatively in addition to the continuing $0.5 \mathrm{mg} /$ week cabergoline. MRI at 3 months postoperatively displayed a significant reduction in the size of the macroadenoma with a small residual suprasellar tumour component surrounding the right carotid artery (Fig. 2B). The optic chiasm was decompressed and showed partial prolapse. Visual field testing demonstrated marked improvement of vision, although some bilateral superior quadrantanopia persisted (Fig. 1D). Serum prolactin was $15 \mathrm{mIU} / \mathrm{L}$ at 4 months postoperatively, and cabergoline was discontinued shortly afterwards due to previous dopamine agonist resistance. Follow-up at 6 months revealed stable visual fields and a normal hormonal profile.

A surveillance MRI 15 months after surgery (Fig. 2C) showed a substantial recurrence of the adenoma with suprasellar and right cavernous sinus extension, along with compression of the optic chiasm. Hyperprolactinaemia $(3,451 \mathrm{mIU} / \mathrm{L})$ was detected, and deterioration of the visual field defect was identified on perimetry. Cabergoline (0.5 mg/week) was re-started pending repeat neurosurgery. Surprisingly, on this occasion, the pituitary lesion showed a remarkable biochemical response together with significant tumour shrinkage. Serum prolactin fell to $20 \mathrm{mIU} / \mathrm{L}$ and repeat pituitary MRI 10 months later 
demonstrated significant shrinkage of the residual tumour with disappearance of the suprasellar component (Fig. 2D). Moreover, visual fields showed considerable improvement. Hence, plans for surgery were suspended, and the patient was followed carefully in the endocrine clinic.

In the interim, we realised that her father, who at the time attended a different endocrinology service, had a metastatic spinal paraganglioma. He was a carrier of a germline missense SDHB mutation (c.298T $>$ C, p.Ser100Pro). This variant, not present in GnomAD database, had previously been identified in a patient with a paraganglioma (13). The father's pituitary gland was found to be normal on MRI imaging. Subsequent genetic testing found the same mutation inherited by our patient. The immunoreaction for SDHB showed complete loss in tumour cells of the father's paraganglioma (Fig. 3D), but retention of the protein in our patient's pituitary adenoma (Fig. 3C). Loss-of-heterozygosity analyses were performed on tissue microdissected from both paraganglioma and pituitary adenoma to enrich the samples with tumour cells and minimise the contamination from normal tissue. Analysis demonstrated partial retention of the normal allele at the mutation site in both tumours.

In light of this evidence, plasma metanephrines and full-body MRI were performed, both of which were normal. A 12-month interval pituitary MRI scan showed a stable appearance. She remains under annual clinical review and biochemical testing (plasma and/or urinary metanephrines), in addition to skull-base to pelvis imaging every $2-3$ years, as recommended by recent literature $(5,6)$.

\section{Discussion}

It is highly unusual for a patient with a germline $S D H B$ mutation to present as an isolated pituitary adenoma. Certainly, germline mutations in $\mathrm{SDHx}$, and more recently $\operatorname{MAX}(7,8)$, are recognised as an infrequent syndromic cause of pituitary adenomas. Whilst screening for paraganglioma/ phaeochromocytoma was negative in our case, this patient remains at increased risk for the development of these tumours in the future. Although the mean age of diagnosis of paraganglioma/phaeochromocytoma in SDHB mutation carriers is reported to be in the third decade $(4,6)$, recent systematic screening of large families identified numerous patients with newly recognised disease at older ages.

Our case adds to the three previous reports of isolated pituitary adenoma in the setting of an inherited $S D H B$ mutation and familial paraganglioma $(2,4)$. All four patients are females, of varying ages at diagnosis. Whilst there is no information available on the size and functional status of one adenoma, the other three (including ours) are macroprolactinomas.

There was an unusual, discordant biochemical and tumour size change response to dopamine receptor agonist therapy in this case. While biochemical response was excellent both initially and at recurrence, the lesion did not shrink after the first treatment, but did when re-challenged after surgery. There is no clear explanation for this unusual response. Our patient's prolactin levels had always been suppressed whilst on treatment suggesting good compliance. One of the two previously reported patients with isolated lactotroph adenoma (2) underwent repeated surgery in addition to radiotherapy, possibly indicating an aggressive tumour behaviour. In addition, LH/FSH-negative, SF-1-positive non-functioning SDHB mutation-positive adenoma following three surgeries and radiotherapy developed metastatic deposits 9 years after the first presentation, and then responded well to temozolomide treatment (9). The limited literature available suggests that pituitary adenomas associated with $S D H x$ mutations exhibit clinically aggressive behaviour, but further data are needed for detailed characterisation of the response to medical treatment $S D H x$-related pituitary adenomas.

Whilst many aspects of the clinical phenotype of $S D H x$-mutated pituitary adenomas remain ill-defined, their unique histological phenotype, as defined by frequent prominent intracytoplasmic vacuoles, is described (2). Intracytoplasmic vacuoles are not commonly encountered in sporadic pituitary adenomas or ones arising in the setting of other genetic mutations, suggesting that their formation could be related to SDH deficiency. They were not described in samples with MAX mutations. The precise mechanism underlying their formation remains to be discovered, though it may be hypothesised that the condition of pseudohypoxia may predispose to the formation of SDH-deficient pituitary adenomas, play a role in their development. As pituitary tumours in $S d h b$ heterozygous mice show increased expression of hypoxia-inducible factor $1 \alpha(H I F-1 \alpha)(10)$, pseudohypoxia could be the basis of SDH-deficient pituitary tumour formation. The presence of prominent intracytoplasmic vacuoles should prompt the examining histopathologist to perform SDHB and SDHA immunostaining to screen for a possible $S D H x$ mutation. A recent multicentre interobserver variation analysis on the use of SDHB/SHDA immunohistochemistry in paragangliomas and phaeochromocytomas and a study on pituitary adenomas confirmed its use as a cheap and reliable tool to identify patients with $S D H x$ mutations, prior to genetic testing $(11,12)$. In our specific case, the substitution p.Ser100Pro might have resulted in impaired SDHB 
binding to iron-sulphur clusters and possibly in impaired interactions between the protein and the other subunits. In in vitro studies, the p.Ser100Pro substitution did not show elevated succinate level, but had an increased succinate/ fumarate ratio (13). Immunohistochemistry for SDHB was not performed by Pollard and colleagues. The anti-SDHB antibody used in our study recognises the portion of SDHB between glutamic acid in position 167 and tyrosine 273 that is downstream of the site of the mutation. It is possible that mutated SDHB becomes less stable and degraded at a higher rate than the WT protein in the paraganglioma tissue but not in the pituitary adenoma. The retention of the normal allele could also explain normal SDHB expression in the pituitary adenoma and raises the question if $S D H B$ haploinsufficiency could be sufficient for tumour formation.

In conclusion, pituitary adenomas may remain the sole manifestation of $S D H B$ mutation-related disease. Furthermore, this case demonstrates that immunohistochemistry is not always predictive of $S D H x$ mutations and therefore cannot replace genetic analysis.

\section{Declaration of interest}

The authors declare that there is no conflict of interest that could be perceived as prejudicing the impartiality of the research reported.

\section{Funding}

N T was supported by The Medical College of Saint Bartholomew's Hospital Trust, J D was supported by a grant from the Brazilian Federal Agency for Support and Evaluation of Post-graduate Education (CAPES). Ongoing support to our endocrine tumour genetics program from the NIHR (National Institute of Health Research, UK) are gratefully acknowledged.

\section{Patient consent}

Written informed consent has been obtained from the patient for publication of the submitted article and accompanying images.

\section{Author contribution statement}

$\mathrm{M} \mathrm{M}, \mathrm{F} \mathrm{R}, \mathrm{M} \mathrm{K}$ and F W wrote the manuscript. F W, N M, N C, M K-S, B B, $A G, K M, N M$ and $F$ R provided diagnostic support and clinical care of the patient, D C, J D and N T performed the loss of heterozygosity studies. All authors reviewed and edited the manuscript.

\section{References}

1 Caimari F \& Korbonits M. Novel genetic causes of pituitary adenomas. Clinical Cancer Research 201622 5030-5042. (https://doi. org/10.1158/1078-0432.CCR-16-0452)

2 Dénes J, Swords F, Rattenberry E, Stals K, Owens M, Cranston T, Xekouki P, Moran L, Kumar A, Wassif C, et al. Heterogeneous genetic background of the association of pheochromocytoma/paraganglioma and pituitary adenoma - results from a large patient cohort. Journal of Clinical Endocrinology and Metabolism 2015100 E531-E541. (https://doi.org/10.1210/jc.2014-3399)

3 Xekouki P, Pacak K, Almeida M, Wassif CA, Rustin P, Nesterova M, de la Luz Sierra M, Matro J, Ball E, Azevedo M, et al. Succinate dehydrogenase (SDH) D subunit (SDHD) inactivation in a growthhormone-producing pituitary tumor: a new association for SDH? Journal of Clinical Endocrinology and Metabolism 201297 E357-E366. (https://doi.org/10.1210/jc.2011-1179)

4 Benn DE, Gimenez-Roqueplo AP, Reilly JR, Bertherat J, Burgess J, Byth K, Croxson M, Dahia PL, Elston M, Gimm O, et al. Clinical presentation and penetrance of pheochromocytoma/paraganglioma syndromes. Journal of Clinical Endocrinology and Metabolism 200691 827-836. (https://doi.org/10.1210/jc.2005-1862)

5 Eijkelenkamp K, Osinga TE, de Jong MM, Sluiter WJ, Dullaart RP, Links TP, Kerstens MN \& van der Horst-Schrivers AN. Calculating the optimal surveillance for head and neck paraganglioma in SDHBmutation carriers. Familial Cancer 201716 123-130. (https://doi. org/10.1007/s10689-016-9923-3)

6 Tufton N, Shapiro L, Srirangalingam U, Richards P, Sahdev A, Kumar AV, McAndrew L, Martin L, Berney D, Monson J, et al. Outcomes of annual surveillance imaging in an adult and paediatric cohort of succinate dehydrogenase B mutation carriers. Clinical Endocrinology 201786 286-296. (https://doi.org/10.1111/cen.13246)

7 Roszko KL, Blouch E, Blake M, Powers JF, Tischler AS, Hodin R, Sadow P \& Lawson EA. Case report of a prolactinoma in a patient with a novel MAX mutation and bilateral pheochromocytomas. Journal of the Endocrine Society 2017 1401-1407.

8 Daly AF, Castermans E, Oudijk L, Guitelman MA, Beckers P, Potorac I, Neggers S, Sacre N, van der Lely AJ, Bours V, et al. Pheochromocytomas and pituitary adenomas in three patients with MAX exon deletions. Endocrine-Related Cancer 201825 L37-L42. (https://doi.org/10.1530/ERC-18-0065)

9 Tufton N, Roncaroli F, Hadjidemetriou I, Dang MN, Denes J, Guasti L, Thom M, Powell M, Baldeweg SE, Fersht N, et al. Pituitary carcinoma in a patient with an SDHB mutation. Endocrine Pathology 201728 320-325.

10 Xekouki P, Szarek E, Bullova P, Giubellino A, Quezado M, Mastroyannis SA, Mastorakos P, Wassif CA, Raygada M, Rentia N, et al. Pituitary adenoma with paraganglioma/pheochromocytoma (3PAs) and succinate dehydrogenase defects in humans and mice. Journal of Clinical Endocrinology and Metabolism 2015100 E710-E719. (https://doi.org/10.1210/jc.2014-4297)

11 Gill AJ, Toon CW, Clarkson A, Sioson L, Chou A, Winship I, Robinson BG, Benn DE, Clifton-Bligh RJ \& Dwight T. Succinate dehydrogenase deficiency is rare in pituitary adenomas. American Journal of Surgical Pathology 201438 560-566. (https://doi. org/10.1097/PAS.0000000000000149)

12 Papathomas TG, Oudijk L, Persu A, Gill AJ, van Nederveen F, Tischler AS, Tissier F, Volante M, Matias-Guiu X, Smid M, et al. SDHB/SDHA immunohistochemistry in pheochromocytomas and paragangliomas: a multicenter interobserver variation analysis using virtual microscopy: a Multinational Study of the European Network for the Study of Adrenal Tumors (ENS@T). Modern Pathology 201528 807-821. (https://doi.org/10.1038/modpathol.2015.41)

13 Pollard PJ, Briere JJ, Alam NA, Barwell J, Barclay E, Wortham NC, Hunt T, Mitchell M, Olpin S, Moat SJ, et al. Accumulation of Krebs cycle intermediates and over-expression of HIF1alpha in tumours which result from germline FH and SDH mutations. Human Molecular Genetics 200514 2231-2239. (https://doi.org/10.1093/ hmg/ddi227)

Received in final form 28 May 2018

Accepted 20 June 2018 